\title{
Eigenvalue approach to nanoscale beam in modified couple stress thermo-elastic diffusion
}

\author{
Rajneesh Kumar ${ }^{a}$ and Shaloo Devi ${ }^{\text {** }}$
}

${ }^{a}$ Department of Mathematics, Kurukshetra University, Kurukshetra, India

${ }^{b}$ Department of Mathematics \& Statistics, Himachal Pradesh University, Shimla, India

\begin{tabular}{l}
\hline A R T I C L EI N F O \\
\hline Article history: \\
Received 6 June, 2017 \\
Accepted 7 September 2017 \\
Available online \\
7 September 2017 \\
\hline Keywords: \\
Modified couple stress theory \\
Thermoelastic diffusion \\
Nanoscale beam \\
Laplace transform \\
Eigen value approach \\
Ramp type heating
\end{tabular}

A B S T R A C T

\begin{abstract}
The problem of thermoelastic nanoscale beam based on a modified couple stress theory with diffusion subjected to ramp type heating is investigated. The Laplace transform technique and eigen value approach are applied to solve the equations which are written in the dimensionless form. The expressions for displacement, lateral deflection, temperature change, mass concentration, axial stress and chemical potential are derived in the transformed domain. A general algorithm of the inverse Laplace transform is developed to compute the results numerically. The mathematical model is prepared for Copper material. The resulting quantities are depicted graphically to show the effects of time. Some particular cases of interest are also deduced from the present problem.
\end{abstract}

(C) 2017 Growing Science Ltd. All rights reserved.

\section{Introduction}

Voigt (1887) was the first who introduced the concept of couple stress linear theory of elasticity and then this theories extended by Cosserat and Cosserat (1909). Couple-stress theory is an extended continuum theory that includes the effects of a couple per unit area on a material volume, in addition to the classical direct and shear forces per unit area. This immediately admits the possibility of asymmetric stress tensor, since shear stress no longer have to be conjugate in order to ensure rotational equilibrium. Toupin (1962) derived the associative constitutive equations for finite deformation of perfectly elastic materials. Mindlin and Tiersten (1962) formulated a linearized theory of couple stress elasticity. Making use of this theory by Mindlin and Tiersten (1962), the effect of couple stresses were studied on surface waves in elastic media and propagation of waves in an elastic layer by Sengupta and Ghosh (1974a, 1974b). Yang et al. (2002) modified the classical couple stress theory and proposed a modified couple-stress model, in which the couple stress tensor is symmetrical and only one material

\footnotetext{
* Corresponding author.

E-mail addresses: shaloosharma2673@gmail.com (S. Devi) 
length parameter is needed to capture the size effect which is caused by micro-structure. Simsek and Reddy (2013) investigated the bending and vibration of functionally graded microbeams using a new higher order beam theory and the modified couple stress theory. Recently, Shaat et al. (2014) studied the size-dependent bending analysis of Kirchhoff nano-plates based on a modified couple-stress theory including surface effects. Samaei et al. (2015), analyzed vibration response of a graphene sheet embedded in an elastic medium and considered the small scale effects in this regard.

Thermo-diffusion is used to describe the processes of thermomechanical treatment of metals (carboning, nitriding steel, etc.) and these processes are thermally activated, and their diffusing substances being, e.g. nitrogen, carbon etc. They are accompanied by deformations of the solid. The theory of thermoelastic with mass diffusion was firstly developed by Nowacki (1974). In this theory, the coupled thermoelastic model is used. This implies infinite speeds of propagation of thermoelastic waves. Sherief et al. (2004) developed the theory of generalized thermoelastic diffusion that predicts finite speeds of propagation for thermoelastic and diffusive waves. Sherief and Saleh (2005) worked on a problem of a thermoelastic half space with a permeating substance in contact with the bounding plane in the context of the theory of generalized thermoelastic diffusion with one relaxation time. The basic equations in generalized thermoelastic diffusion for Green Lindsay (GL-model) theory was derived by Kumar and Kansal (2008).

The significance of using eigenvalue approach to reduce the problem on vector-matrix differential equation to an algebraic eigenvalue problems. Thus the solutions for the field variables are obtained by determining the eigenvalues and the corresponding eigenvectors. In this approach, the physical quantities are directly involved in the formulation of the problem and as such the boundary and initial conditions can be applied directly. Choudhary and Deswal (2010) studied the two-dimensional problem of generalized thermoelastic diffusion using the eigenvalue approach. Sarkar and Lahiri (2012) discussed the two-temperature magnetothermoelastic problem in the context of one relaxation time. The Laplace transform and eigenvalue approach are used to solve the basic equations. Zang and Fu (2012) developed a new beam model for a viscoelastic micro-beam based on a modified couple stress theory. Rezazadeh et al. (2012) discussed problem of thermoelastic damping in a micro-beam resonator using modified couple stress theory. Abouelregal and Zenkour (2014) discussed the problem of an axially moving microbeam subjected to sinusoidal pulse heating and an external transverse excitation with one relaxation time by using Laplace transform and also studied the effects of the pulse-width of thermal vibration, moving speed and the transverse excitation. Yong et al. (2015) presented a nonlinear bending and post-buckling of extensible microscale beams based on modified couple stress theory where the effects of the material length scale parameter and the Poisson ratio on the bending and thermal post-buckling behaviors of microbeams are discussed in detail. Kumar and Devi (2015) presented the problem of hall current and rotation in a modified couple stress theory due to ramp type loading. Thermoelastic interaction in a thermally conducting cubic crystal subjected to ramp-type heating was investigated by Abbas et al. (2015). Reddy et al. (2016) discussed the problem of functionally graded circular plates with modified couple stress theory by using finite element method. On the basis of global local theory, a model for a composite laminated Reddy plate of new modified couple-stress theory was developed by Chen and Wang (2016). Zenkour and Abouelregal (2016) discussed the vibration of functionally graded microbeams by using Green-Naghdi thermoelasticity theory (1993) and Laplace transform.

In this work, the governing equations of the modified couple stress theory of thermoelastic beam induced by ramp-type heating is developed. The basic equations are solved by applying Laplace transform technique and eigenvalue approach. Analytical method is used to determine the lateral deflection, displacement, temperature change, mass concentration, chemical potential and axial stress of the beam. The effects of time on the field quantities are studied and shown graphically. 


\section{Basic equations}

The constitutive relations, equations of motion, equation of heat conduction, equation of mass diffusion in a modified couple-stress generalized thermoelastic with mass diffusion in the absence of body forces, body couples, heat and mass diffusive sources are given by Yang et al. (2002), Sherief et al. (2004).

$$
\begin{aligned}
& t_{i j}=\lambda e \delta_{i j}+2 \mu e_{i j}-\frac{1}{2} e_{k i j} m_{l k, l}-\beta_{1} T \delta_{i j}-\beta_{2} C \delta_{i j}, \\
& m_{i j}=2 \alpha \chi_{i j} \\
& \chi_{i j}=\frac{1}{2}\left(\omega_{i, j}+\omega_{j, i}\right), i, j, k=1,2,3 . \\
& \omega_{i}=\frac{1}{2} e_{i p q} u_{q, p}, \\
& P=-\beta_{2} e_{k k}-a T+b C \\
& \left(\lambda+\mu+\frac{\alpha}{4} \Delta\right) \nabla(\nabla \cdot \boldsymbol{u})+\left(\mu-\frac{\alpha}{4} \Delta\right) \nabla^{2} \boldsymbol{u}-\beta_{1} \nabla T-\beta_{2} \nabla C=\rho \ddot{\boldsymbol{u}}, \\
& K \Delta T-\left(\frac{\partial}{\partial t}+\tau_{0} \frac{\partial^{2}}{\partial t^{2}}\right)\left(\rho c_{e} T+a T_{0} C+T_{0} \beta_{1}(\nabla \cdot \boldsymbol{u})\right)=0, \\
& D \beta_{2} \Delta(\nabla \cdot \boldsymbol{u})+D a \Delta T-D b \Delta C+\left(\frac{\partial}{\partial t}+\tau_{1} \frac{\partial^{2}}{\partial t^{2}}\right) C=0,
\end{aligned}
$$

where $t_{i j}$ are the components of stress tensor, $\lambda$ and $\mu$ are material constants, $\delta_{i j}$ is Kronecker's delta, $e_{i j}$ are the components of strain tensor, $e_{i j k}$ is alternate tensor, $m_{i j}$ are the components of couple-stress, $\beta_{1}=(3 \lambda+2 \mu) \alpha_{t}, \beta_{2}=(3 \lambda+2 \mu) \alpha_{c}$, Here $\alpha_{t}, \alpha_{c}$ are the coefficients of linear thermal expansion and diffusion expansion respectively, $T$ is the temperature change, $C$ is the mass concentration, $\alpha$ is the couple stress parameter, $\chi_{i j}$ is symmetric curvature, $\omega_{i}$ is the rotational vector, $P$ is the chemical potential of the material per unit mass, $b$ is the coefficient describing the measure of mass diffusion effects, $a$ is the coefficient describing the measure of thermoelastic diffusion. $\boldsymbol{u}$ is the displacement vector, $\rho$ is the density, $\Delta$ is the Laplacian operator, $\nabla$ is del operator. $K$ is the coefficient of the thermal conductivity, $c_{e}$ is the specific heat at constant strain, $T_{0}$ is the reference temperature assumed to be such that $T / T_{0}<<1 . D$ is the thermoelastic diffusion constant, Here $\tau_{0}$ are thermal relaxation time and $\tau_{1}$ are the diffusion relaxation time.

\section{Formulation of the problem}

We consider a homogeneous, isotropic, rectangular modified couple stress thermoelastic-diffusive beam with dimensions of length $(0 \leq x \leq L)$, width $(-d / 2 \leq y \leq d / 2)$ and thickness $(-h / 2 \leq z \leq h / 2)$ as shown in Fig. 1. We define the $x$-axis along the length of the beam, and the $y$-axis along the width and $z$-axes along the thickness which also represent the axis of material symmetry. Thus, any plane cross-section initially perpendicular to the axis of the beam remains plane and perpendicular to the neutral surface during bending. 


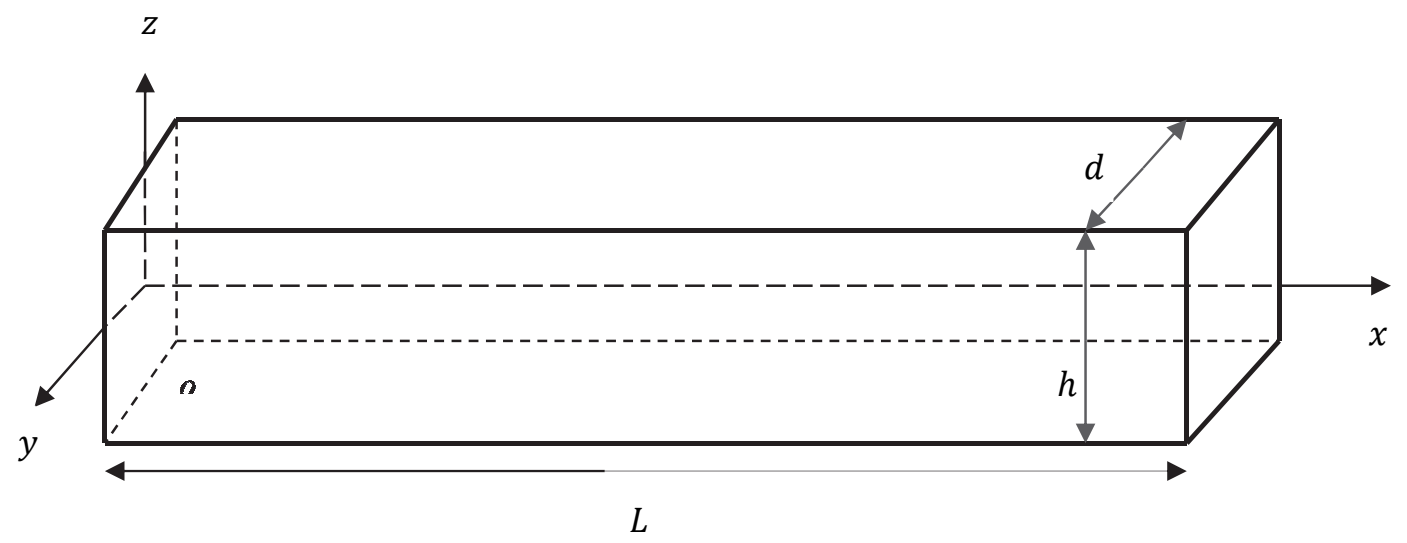

Fig. 1. Problem Description

According to the fundamental Euler-Bernoulli theory for small deflection of a simple bending problem, the displacement components are given by

$$
u=-z \frac{\partial w}{\partial x}, v=0, w(x, y, z, t)=w(x, t)
$$

where $w(x, t)$ is the lateral deflection of the beam and $t$ is the time. The constitutive Eq. (1) in onedimension along the axis and with the help of Eq. (9), we obtain

$$
t_{x}=-(\lambda+2 \mu) z \frac{\partial^{2} w}{\partial x^{2}}-\beta_{1} T-\beta_{2} C
$$

Then the bending moment of the cross-section of the beam is given by

$$
M=M_{\sigma}+M_{m}=d\left(\int_{\frac{-h}{2}}^{\frac{h}{2}} t_{x} z d z+\int_{\frac{-h}{2}}^{\frac{h}{2}} m_{x y} d z\right),
$$

where $M_{\sigma}$ and $M_{m}$ are the components of the bending moment due to the classic stress and couple stress tensors respectively.

Using Euler-Bernoulli assumption (9) and with the aid of Eq. (10) in Eq. (11), yields

$$
M=-(\lambda+2 \mu) I \frac{\partial^{2} w}{\partial x^{2}}-M_{T}-M_{C}-\alpha A \frac{\partial^{2} w}{\partial x^{2}},
$$

where $I$ is the second moment of the cross-section area of the beam, $M_{T}$ is the thermal moment, $M_{C}$ is the mass moments of the beam. Thus, $I, M_{T}$ and $M_{C}$ are given by

$$
I=\int_{\frac{-h}{2}}^{\frac{h}{2}} d z^{2} d z=\frac{d h^{3}}{12}, M_{T}=\beta_{1} d \int_{\frac{-h}{2}}^{\frac{h}{2}} T z d z, M_{C}=\beta_{2} d \int_{\frac{-h}{2}}^{\frac{h}{2}} C z d z .
$$


The equation of transverse motion of the beam is given by Rao (2007):

$$
\frac{\partial^{2} M}{\partial x^{2}}-\rho A \frac{\partial^{2} w}{\partial t^{2}}=0
$$

where $\rho$ denotes the beam density and $A=d h$ is the cross -sectional area of the beam.

Substituting Eqs. (12) in Eq. (14), we obtain

$$
[(\lambda+2 \mu) I+\alpha A] \frac{\partial^{4} w}{\partial x^{4}}+\frac{\partial^{2} M_{T}}{\partial x^{2}}+\frac{\partial^{2} M_{C}}{\partial x^{2}}+\rho A \frac{\partial^{2} w}{\partial t^{2}}=0 .
$$

The heat conduction equation and the mass diffusion equation can be written as

$$
\begin{aligned}
& K\left(\frac{\partial^{2} T}{\partial x^{2}}+\frac{\partial^{2} T}{\partial z^{2}}\right)-\left(\frac{\partial}{\partial t}+\tau_{0} \frac{\partial^{2}}{\partial t^{2}}\right)\left(\rho c_{e} T+a T_{0} C-\beta_{1} T_{0} z \frac{\partial^{2} w}{\partial x^{2}}\right)=0 \\
& D \beta_{2} z \frac{\partial^{4} w}{\partial x^{4}}-D a\left(\frac{\partial^{2} T}{\partial x^{2}}+\frac{\partial^{2} T}{\partial z^{2}}\right)-\left(\frac{\partial}{\partial t}+\tau_{1} \frac{\partial^{2}}{\partial t^{2}}\right) C+D b\left(\frac{\partial^{2} T}{\partial x^{2}}+\frac{\partial^{2} T}{\partial z^{2}}\right)=0 .
\end{aligned}
$$

For a very thin beam, assuming the temperature increment and mass concentration varies in terms of $\sin (p z) \quad$ function along the thickness of the beam.

$$
T(x, z, t)=T_{1}(x, t) \sin (p z), \quad C(x, z, t)=C_{1}(x, t) \sin (p z)
$$

where $p=\pi / h$.

Multiplying Eq. (16) and Eq. (17) by $z d z$ and integrate over the interval $(-h / 2, h / 2)$, we obtain

$$
\begin{aligned}
& \frac{\partial^{2} T_{1}}{\partial x^{2}}-p^{2} T_{1}-\left(\frac{\partial}{\partial t}+\tau_{0} \frac{\partial^{2}}{\partial t^{2}}\right)\left[\frac{\rho c_{e}}{K} T_{1}+\frac{a T_{0}}{K} C_{1}-\frac{\beta_{1} T_{0} \pi^{2} h}{24 K} \frac{\partial^{2} w}{\partial x^{2}}\right]=0 \\
& \frac{D \beta_{2} \pi^{2} h}{24}\left(\frac{\partial^{4} w}{\partial x^{4}}\right)-D a\left(\frac{\partial^{2} T_{1}}{\partial x^{2}}-p^{2} T_{1}\right)-\left(\frac{\partial}{\partial t}+\tau_{1} \frac{\partial^{2}}{\partial t^{2}}\right) C_{1}+D b\left(\frac{\partial^{2} C_{1}}{\partial x^{2}}-p^{2} C_{1}\right)=0
\end{aligned}
$$

For convenience, we define the following dimensionless quantities:

$$
\begin{aligned}
& \left(x^{\prime}, z^{\prime}, u^{\prime}, w^{\prime}\right)=\frac{(x, z, u, w)}{L}, \tau_{0}^{\prime}=\frac{v \tau_{0}}{L}, \tau_{1}^{\prime}=\frac{v \tau_{1}}{L}, t^{\prime}=\frac{v t}{L}, T^{\prime}=\frac{T}{T_{0}}, C^{\prime}=\frac{C}{C_{0}}, P^{\prime}=\frac{P}{b C_{0}}, \\
& M^{\prime}=\frac{M}{d E h^{2}}, M_{T}^{\prime}=\frac{M_{T}}{d E h^{2}}, M_{C}^{\prime}=\frac{M_{C}}{d E h^{2}}, t_{x}^{\prime}=\frac{t_{x}}{E}, v^{2}=\frac{E}{\rho} .
\end{aligned}
$$

Using Eqs. (18) and Eqs. (21), with the aid of Eq. (13) in Eq. (15), Eq. (19) and Eq. (20), after dropping the dashes for convenience, yield

$$
\frac{\partial^{4} w}{\partial x^{4}}+a_{1}\left(\frac{\partial^{2} T_{1}}{\partial x^{2}}\right)+a_{2}\left(\frac{\partial^{2} C_{1}}{\partial x^{2}}\right)+a_{3} \frac{\partial^{2} w}{\partial t^{2}}=0
$$




$$
\begin{aligned}
& \frac{\partial^{2} T_{1}}{\partial x^{2}}-a_{4} T_{1}-\left(\frac{\partial}{\partial t}+\tau_{0} \frac{\partial^{2}}{\partial t^{2}}\right)\left[a_{5} T_{1}+a_{6} C_{1}-a_{7} \frac{\partial^{2} w}{\partial x^{2}}\right]=0 \\
& \frac{\partial^{2} C_{1}}{\partial x^{2}}-a_{4} C_{1}+a_{8}\left(\frac{\partial^{4} w}{\partial x^{4}}\right)-a_{9}\left(\frac{\partial^{2} T_{1}}{\partial x^{2}}-a_{3} T_{1}\right)-a_{10}\left(\frac{\partial}{\partial t}+\tau_{1} \frac{\partial^{2}}{\partial t^{2}}\right) C_{1}=0,
\end{aligned}
$$

where

$$
\begin{aligned}
& a_{1}=\frac{2 d \beta_{1} T_{0} L}{p^{2}[(\lambda+2 \mu) I+\alpha A]}, a_{2}=\frac{2 d \beta_{2} C_{0} L}{p^{2}[(\lambda+2 \mu) I+\alpha A]}, a_{3}=\frac{\rho A v^{2} L^{2}}{[(\lambda+2 \mu) I+\alpha A]}, a_{4}=p^{2} L^{2}, \\
& a_{5}=\frac{\rho c_{e} v L}{K}, a_{6}=\frac{a C_{0} v L}{K}, a_{7}=\frac{v \beta_{1} \pi^{2} h}{24 K}, a_{8}=\frac{\beta_{2} \pi^{2} h}{24 L b C_{0}}, a_{9}=\frac{a T_{0}}{b C_{0}}, a_{10}=\frac{v L}{D b}
\end{aligned}
$$

\section{Solution of the Problem}

We define the Laplace transform as

$$
L\{f(t)\}=\int_{0}^{\infty} e^{-s t} f(t) d t=\bar{f}(s)
$$

where $S$ is the Laplace transform parameter. Making use of relation (25) in Eqs. (22-24), we obtain:

$$
\begin{aligned}
& \frac{d^{4} \bar{w}}{d x^{4}}+a_{1}\left(\frac{d^{2} \bar{T}_{1}}{d x^{2}}\right)+a_{2}\left(\frac{d^{2} \bar{C}_{1}}{d x^{2}}\right)+a_{3} s^{2} \bar{w}=0, \\
& \frac{d^{2} \bar{T}_{1}}{d x^{2}}-a_{4} \bar{T}_{1}-\left(s+\tau_{0} s^{2}\right)\left[a_{5} \bar{T}_{1}+a_{6} \bar{C}_{1}-a_{7} \frac{d^{2} \bar{w}}{d x^{2}}\right]=0, \\
& \frac{d^{2} \bar{C}_{1}}{d x^{2}}-a_{4} \bar{C}_{1}=-a_{8}\left(\frac{d^{4} \bar{w}}{d x^{4}}\right)+a_{9}\left(\frac{d^{2} \bar{T}_{1}}{d x^{2}}-a_{3} \bar{T}_{1}\right)+a_{10}\left(s+\tau_{1} s^{2}\right) \bar{C}_{1} .
\end{aligned}
$$

The set of Eqs. (26-28) can be written as

$$
\begin{aligned}
& \frac{d^{2} \bar{w}}{d x^{2}}=a_{52} \bar{v} \\
& \frac{d^{2} \bar{v}}{d x^{2}}=a_{61} \bar{w}+a_{62} \bar{v}+a_{63} \bar{T}_{1}+a_{64} \bar{C}_{1}, \\
& \frac{d^{2} \bar{T}_{1}}{d x^{2}}=a_{72} \bar{v}+a_{73} \bar{T}_{1}+a_{74} \bar{C}_{1}, \\
& \frac{d^{2} \bar{C}_{1}}{d x^{2}}=a_{81} \bar{w}+a_{82} \bar{v}+a_{83} \bar{T}_{1}+a_{84} \bar{C}_{1}, \\
& \text { where } \\
& \tau_{t}^{1}=\left(s+\tau_{0} s^{2}\right), \tau_{t}^{2}=\left(s+\tau_{1} s^{2}\right), \\
& a_{52}=1, a_{61}=\frac{a_{3} s^{2}}{\left(a_{2} a_{8}-1\right)}, a_{62}=\frac{-a_{7}\left(a_{1}+a_{2} a_{9}\right) \tau_{t}^{1}}{\left(a_{2} a_{8}-1\right)}, a_{63}=\frac{a_{1}\left\{a_{4}+a_{5} \tau_{t}^{1}\right\}+a_{2} a_{5} a_{9} \tau_{t}^{1}}{\left(a_{2} a_{8}-1\right)},
\end{aligned}
$$




$$
\begin{aligned}
& a_{64}=\frac{a_{1} a_{6} \tau_{t}^{1}+a_{2}\left\{a_{4}+a_{6} a_{9} \tau_{t}^{1}+a_{10} \tau_{t}^{2}\right\}}{\left(a_{2} a_{8}-1\right)}, a_{72}=-a_{7} \tau_{t}^{1}, a_{73}=a_{4}+a_{5} \tau_{t}^{1}, a_{74}=a_{6} \tau_{t}^{1}, a_{81}=\frac{a_{3} a_{8} s^{2}}{\left(1-a_{2} a_{8}\right)}, \\
& a_{82}=\frac{a_{7} a_{8}\left(a_{1}+a_{2} a_{9}\right) \tau_{t}^{1}}{\left(a_{2} a_{8}-1\right)}, a_{83}=\frac{a_{8}\left[a_{1}\left\{a_{4}+a_{5} \tau_{t}^{1}\right\}+a_{2} a_{5} a_{9} \tau_{t}^{1}\right]}{\left(1-a_{2} a_{8}\right)}, a_{84}=\frac{a_{8}\left[a_{1} a_{6} \tau_{t}^{1}+a_{2}\left\{a_{4}+a_{6} a_{9} \tau_{t}^{1}+a_{10} \tau_{t}^{2}\right\}\right]}{\left(1-a_{2} a_{8}\right)} .
\end{aligned}
$$

The set of Eqs. (29-32) can be written in a matrix form as

$$
D V(x, s)=A V(x, s),
$$

where

$$
V=\left[\begin{array}{c}
U \\
D U
\end{array}\right], U=\left[\begin{array}{llll}
\bar{w} & \bar{v} & \bar{T}_{1} & \bar{C}_{1}
\end{array}\right]^{T}, A=\left[\begin{array}{cc}
O & I \\
A_{1} & O
\end{array}\right], A_{1}=\left[\begin{array}{cccc}
0 & a_{52} & 0 & 0 \\
a_{61} & a_{62} & a_{63} & a_{64} \\
0 & a_{72} & a_{73} & a_{74} \\
a_{81} & a_{82} & a_{83} & a_{84}
\end{array}\right] .
$$

and $I$ is the identity matrix of order 4, $O$ is null matrix of order 4 and []$^{T}$ is the transpose of matrix, $D=\frac{d}{d z}$. To solve the Eq. (33) by the eigenvalue approach as Das et al. (1997). The characteristic equation of the matrix $A$ is given by

$$
\lambda^{8}-G_{1} \lambda^{6}+G_{2} \lambda^{4}-G_{3} \lambda^{2}+G_{4}=0,
$$

where

$$
\begin{aligned}
& G_{1}=a_{62}+a_{73}+a_{84}, G_{2}=a_{84}\left(a_{73}+a_{62}\right)-a_{74} a_{83}-a_{64} a_{82}-a_{63} a_{72}+a_{62} a_{73}+a_{52} a_{61}, \\
& G_{3}=a_{62}\left(a_{74} a_{83}-a_{73} a_{84}\right)-a_{63}\left(a_{74} a_{82}-a_{72} a_{84}\right)+a_{64}\left(a_{73} a_{82}-a_{72} a_{83}\right)+a_{52}\left\{a_{61}\left(a_{73}-a_{84}\right)+a_{64} a_{81}\right\}, \\
& G_{4}=a_{52}\left\{a_{61}\left(a_{73} a_{84}-a_{83} a_{74}\right)+a_{81}\left(a_{63} a_{74}-a_{64} a_{73}\right)\right\} .
\end{aligned}
$$

The characteristic roots of the equation (35), which are also the eigenvalues of the matrix $A$. The eigenvectors $X(x, s)$ corresponding to eigenvalue $\lambda_{r}$ can be determined by solving the homogeneous equations

$$
[A-\lambda I] X(x, s)=0 .
$$

The set of eigen vectors $X_{r}(x, s)$ may be obtained as

$$
X_{r}(x, s)=\left[\begin{array}{c}
X_{r 1}(x, s) \\
X_{r 2}(x, s)
\end{array}\right], \quad X_{r 1}(x, s)=\left[\begin{array}{c}
b_{r} \\
c_{r} \\
d_{r} \\
q_{r}
\end{array}\right], X_{r 2}(x, s)=\lambda_{r} X_{r 1}(x, s) \text { for } \lambda=\lambda_{r}, \quad r=1,2,3,4,
$$

and 


$$
\begin{aligned}
& X_{j}(x, s)=\left[\begin{array}{l}
X_{j 1}(x, s) \\
X_{j 2}(x, s)
\end{array}\right], X_{j 1}(x, s)=\left[\begin{array}{l}
b_{r} \\
c_{r} \\
d_{r} \\
q_{r}
\end{array}\right], X_{j 2}(x, s)=\lambda_{j} X_{j 1}(x, s) \text { for } j=r+4, \lambda=-\lambda_{r}, \quad r=1,2,3,4, \\
& b_{r}=\left[a_{64} a_{83}-a_{63}\left(a_{84}-\lambda_{r}^{2}\right)\right], c_{r}=\lambda_{r}^{2} a_{52}\left[a_{64} a_{83}-a_{63}\left(a_{84}-\lambda_{r}^{2}\right)\right], \\
& d_{r}=a_{52}\left(a_{84}-\lambda_{r}^{2}\right)\left\{a_{52} a_{61}+\lambda_{r}^{2}\left(a_{62}-\lambda_{r}^{2}\right)\right\}-a_{64} a_{52}\left(a_{52} a_{81}+a_{82} \lambda_{r}^{2}\right), \\
& q_{r}=a_{52} a_{63}\left(a_{52} a_{81}+a_{82} \lambda_{r}^{2}\right)-a_{52} a_{83}\left\{a_{52} a_{61}+\lambda_{r}^{2}\left(a_{62}-\lambda_{r}^{2}\right)\right\} .
\end{aligned}
$$

The solution of Eq. (33) can be written as

$$
V=\sum_{r=1}^{4} B_{r} X_{r}(x, s) e^{-\lambda_{r} x}+\sum_{r=1}^{4} B_{r+4} X_{r+4}(x, s) e^{\lambda_{r} x}
$$

where $B_{1}, B_{2}, B_{3}, B_{4}, B_{5}, B_{6}, B_{7}$ and $B_{8}$ are arbitrary constants. Thus, the physical quantities are given by

$$
\left(\bar{w}, \bar{v}, \bar{T}_{1}, \bar{C}_{1}\right)(x, s)=\sum_{r=1}^{4}\left(b_{r}, c_{r}, d_{r}, q_{r}\right) B_{r} e^{-\lambda_{r} x}+\sum_{j=1}^{4}\left(b_{j+4}, c_{j+4}, d_{j+4}, q_{j+4}\right) B_{j+4} e^{\lambda_{j} x} .
$$

\section{Initial and Boundary Conditions}

The initial and boundary conditions should be considered to solve the problem. The initial conditions of the problem are taken in the form as

$$
\left.w(x, t)\right|_{t=0}=\left.\frac{\partial w(x, t)}{\partial t}\right|_{t=0}=0,\left.T(x, t)\right|_{t=0}=\left.\frac{\partial T(x, t)}{\partial t}\right|_{t=0}=0,\left.C(x, t)\right|_{t=0}=\left.\frac{\partial C(x, t)}{\partial t}\right|_{t=0}=0 .
$$

Let us consider a nanobeam with both ends are simply supported:

$$
w(0, t)=0, \frac{\partial^{2} w(0, t)}{\partial x^{2}}=0, w(L, t)=0, \quad \frac{\partial^{2} w(L, t)}{\partial x^{2}}=0 .
$$

We consider the side of the nanobeam $x=0$ is loaded thermally by ramp-type heating, while there is no variation of mass concentration on it. Thus

$$
\begin{aligned}
& T(0, t)=g_{0}\left\{\begin{array}{lr}
0 & t \leq 0, \\
\frac{t}{t_{0}} & 0<t \leq t_{0}, \\
1 & t>t_{0},
\end{array}\right. \\
& \frac{\partial C(0, t)}{\partial x}=0,
\end{aligned}
$$

where $t_{0}$ is a non-negative constant called the ramp type parameter and $g_{0}$ is a constant.

We also assume that the other side of nanobeam $x=L$ is thermally insulated and this means that the following relation will be satisfied: 


$$
\frac{\partial T(L, t)}{\partial x}=0, \frac{\partial C(L, t)}{\partial x}=0
$$

Making use of Eq. (18), Eq. (21) and Eq. (25) in the boundary conditions (40-43), we obtain:

$$
\begin{aligned}
& \bar{w}(0, s)=0, \quad \frac{d^{2} \bar{w}(0, s)}{d x^{2}}=0, \quad \bar{T}_{1}(0, s)=g_{0}\left(\frac{1-e^{-s t_{0}}}{t_{0} s^{2}}\right), \frac{d \bar{C}_{1}(0, s)}{d x}=0 . \\
& \bar{w}(1, s)=0, \frac{d^{2} \bar{w}(1, s)}{d x^{2}}=0, \frac{d \bar{T}_{1}(1, s)}{d x}=0, \frac{d \bar{C}_{1}(1, s)}{d x}=0 .
\end{aligned}
$$

The values of $\bar{u}, \overline{t_{x}}$ and $\bar{P}$ are given by

$$
\begin{aligned}
& \bar{u}(x, s)=z\left[\sum_{i=1}^{4} \lambda_{i} b_{i} B_{i} e^{-\lambda_{i} x}-\sum_{i=1}^{4} \lambda_{i} b_{i+4} B_{i+4} e^{\lambda_{i} x}\right], \\
& \bar{t}_{x}(x, s)=-\left[\sum_{i=1}^{4}\left\{\frac{(\lambda+2 \mu)}{E} z \lambda_{i}^{2} b_{i}+\sin (p z)\left(\frac{\beta_{1} T_{0}}{E} d_{i}+\frac{\beta_{2} C_{0}}{E} q_{i}\right)\right\} B_{i} e^{-\lambda_{i} x}+\sum_{i=1}^{4}\left\{\begin{array}{l}
\frac{(\lambda+2 \mu)}{E} z \lambda_{i}^{2} b_{i+4} \\
+\sin (p z)\left(\frac{\beta_{1} T_{0}}{E} d_{i+4}+\frac{\beta_{2} C_{0}}{E} q_{i+4}\right)
\end{array}\right\} B_{i+4} e^{\lambda_{i} x}\right], \\
& \bar{P}(x, s)=\left[\sum_{i=1}^{4}\left\{z a_{11} \lambda_{i}^{2} b_{i}-\sin (p z)\left(a_{9} d_{i}-q_{i}\right)\right\} B_{i} e^{-\lambda_{i} x}+\sum_{i=1}^{4}\left\{\begin{array}{l}
z a_{11} \lambda_{i}^{2} b_{i+4} \\
-\sin (p z)\left(a_{9} d_{i+4}-q_{i+4}\right)
\end{array}\right\} B_{i+4} e^{\lambda_{i} x}\right],
\end{aligned}
$$

where

$a_{11}=\frac{\beta_{2}}{b C_{0}}$.

Substituting Eq. (38) and Eqs. (46-48) in the boundary conditions (44) and (45), after some simplification, we obtain the expressions of displacement, lateral deflection, temperature change, mass concentration, axial stress and chemical potential of the beam as

$$
\left(\bar{u}, \bar{w}, \bar{T}, \bar{C}_{1}, \bar{t}_{x}, \bar{P}\right)(x, s)=\sum_{i=1}^{4}\left\{\left(z \lambda_{i}+1\right) b_{i}, d_{i}, q_{i}, M_{i}, N_{i}\right\} B_{i} e^{-\lambda_{i} x}-\sum_{i=1}^{4}\left\{\left(z \lambda_{i+4}+1\right) b_{i+4}, d_{i+4}, q_{i+4}, M_{i+4}, N_{i+4}\right\} B_{i+4} e^{\lambda_{i} x}
$$

where

$$
\begin{aligned}
& \sum_{j=1}^{8} B_{j}=\sum_{j=1}^{8} \frac{\Delta_{j}}{\Delta} \text {. } \quad \sum_{i=1}^{4} M_{i}=-\left\{\frac{(\lambda+2 \mu)}{E} z \lambda_{i}^{2} b_{i}+\sin (p z)\left(\frac{\beta_{1} T_{0}}{E} d_{i}+\frac{\beta_{2} C_{0}}{E} q_{i}\right)\right\}, \\
& \sum_{i=1}^{4} M_{i+4}=-\left\{\frac{(\lambda+2 \mu)}{E} z \lambda_{i}^{2} b_{i+4}+\sin (p z)\left(\frac{\beta_{1} T_{0}}{E} d_{i+4}+\frac{\beta_{2} C_{0}}{E} q_{i+4}\right)\right\}, \\
& \sum_{i=1}^{4} N_{i}=\left\{a_{11} z \lambda_{i}^{2} b_{i}-\sin (p z)\left(a_{9} d_{i}-q_{i}\right)\right\}, \sum_{i=1}^{4} N_{i+4}=\left\{a_{11} z \lambda_{i}^{2} b_{i+4}-\sin (p z)\left(a_{9} d_{i+4}-q_{i+4}\right)\right\} .
\end{aligned}
$$


$\Delta=\left[\begin{array}{cccccccc}b_{1} & b_{2} & b_{3} & b_{4} & b_{5} & b_{6} & b_{7} & b_{8} \\ b_{1} e^{-\lambda_{1}} & b_{2} e^{-\lambda_{2}} & b_{3} e^{-\lambda_{3}} & b_{4} e^{-\lambda_{4}} & b_{5} e^{\lambda_{1}} & b_{6} e^{\lambda_{2}} & b_{7} e^{\lambda_{3}} & b_{8} e^{\lambda_{4}} \\ b_{1} \lambda_{1}^{2} & b_{2} \lambda_{2}^{2} & b_{3} \lambda_{3}^{2} & b_{4} \lambda_{4}^{2} & b_{5} \lambda_{1}^{2} & b_{6} \lambda_{2}^{2} & b_{7} \lambda_{3}^{2} & b_{8} \lambda_{4}^{2} \\ b_{1} \lambda_{1}^{2} e^{-\lambda_{1}} & b_{2} \lambda_{2}^{2} e^{-\lambda_{2}} & b_{3} \lambda_{3}^{2} e^{-\lambda_{3}} & b_{4} \lambda_{4}^{2} e^{-\lambda_{4}} & b_{5} \lambda_{1}^{2} e^{\lambda_{1}} & b_{6} \lambda_{2}^{2} e^{\lambda_{2}} & b_{7} \lambda_{3}^{2} e^{\lambda_{3}} & b_{8} \lambda_{4}^{2} e^{\lambda_{4}} \\ d_{1} & d_{2} & d_{3} & d_{4} & d_{5} & d_{6} & d_{7} & d_{8} \\ -d_{1} \lambda_{1} e^{-\lambda_{1}} & -d_{2} \lambda_{2} e^{-\lambda_{2}} & -d_{3} \lambda_{3} e^{-\lambda_{3}} & -d_{4} \lambda_{4} e^{-\lambda_{4}} & d_{5} \lambda_{1} e^{\lambda_{1}} & d_{6} \lambda_{2} e^{\lambda_{2}} & d_{7} \lambda_{7} e^{\lambda_{3}} & d_{8} \lambda_{8} e^{\lambda_{4}} \\ -q_{1} \lambda_{1} & -q_{2} \lambda_{2} & -q_{3} \lambda_{3} & -q_{4} \lambda_{4} & q_{5} \lambda_{1} & q_{6} \lambda_{2} & q_{7} \lambda_{3} & q_{8} \lambda_{4} \\ -q_{1} \lambda_{1} e^{-\lambda_{1}} & -q_{2} \lambda_{2} e^{-\lambda_{2}} & -q_{3} \lambda_{3} e^{-\lambda_{3}} & -q_{4} \lambda_{4} e^{-\lambda_{4}} & q_{5} \lambda_{1} e^{\lambda_{1}} & q_{6} \lambda_{2} e^{\lambda_{2}} & q_{7} \lambda_{3} e^{\lambda_{3}} & q_{8} \lambda_{4} e^{\lambda_{4}}\end{array}\right]$

$\Delta_{i}(i=1, \ldots \ldots \ldots \ldots, 8)$ are obtain by replacing $1^{\text {st }}, 2^{\text {nd }}, 3^{\text {rd }}, 4^{\text {th }}, 5^{\text {th }}, 6^{\text {th }}, 7^{\text {th }}$ and $8^{\text {th }}$ column by $\left[0,0,0,0, g_{0}\left(\frac{1-e^{-s t_{0}}}{t_{0} s^{2}}\right), 0,0,0\right]^{T}$ in $\Delta_{i}$.

\subsection{Particular Cases}

(i) If $\alpha=0$, in Eqs. (49), we obtain the results for displacement, lateral deflection, temperature change, mass concentration, chemical potential and axial stress of the beam for thermoelastic beam with mass diffusion by the use of eigenvalue approach and these results in a special case are similar as obtained by Alzahrani and Abbas (2015).

(ii) In the absence of diffusion $\left(\beta_{2}=a=\tau_{t}^{2}=0\right)$, in Eqs. (49), we obtain the corresponding expressions for displacement, lateral deflection, temperature change, mass concentration, chemical potential and axial stress in a modified couple stress thermoelastic beam by using the eigenvalue approach.

(iii) If we take $\tau_{0}=\tau_{1}=0$, in Eqs. (49), we obtain the corresponding results for modified couple stress thermoelastic diffusion with eigenvalue approach for Coupled thermoelastic (CT) model.

\section{Inversion of the Laplace transform}

We obtained the solutions for the displacement, lateral deflection, temperature change, mass concentration, axial stress and chemical potential in the Laplace transform domain $(x, s)$. We shall now outline briefly the numerical inversion method used to find the solution in the physical domain. Let $\bar{f}(s)$ be the Laplace transform of a function $f(t)$. The inversion formula of Laplace transform can be written as

$$
\begin{aligned}
& \bar{f}(s)=L[f(t)]=\int_{0}^{\infty} e^{-s t} f(t) d t, \\
& f(t)=L^{-1}[\bar{f}(s)]=\frac{1}{2 \pi i} \int_{c-i \infty}^{c+i \infty} e^{s t} \bar{f}(s) d s,
\end{aligned}
$$

with $s=c+i g$;

where $c$ is an arbitrary real number greater than all the real parts of the singularities of $\bar{f}(s)$. We adopt a numerical inversion method on the Fourier series expansion, by which the integral (51) can be approximated as a series 


$$
\begin{aligned}
f(t)= & \frac{e^{c t}}{t_{1}}\left[-\frac{1}{2} \operatorname{Re} \bar{f}(c)+\sum_{j=0}^{\infty} \operatorname{Re}\left(\bar{f}\left(c+\frac{i j \pi}{t_{1}}\right)\right) \cos \left(\frac{j \pi}{t_{1}}\right)-\sum_{j=0}^{\infty} \operatorname{Im}\left(\bar{f}\left(c+\frac{i j \pi}{t_{1}}\right)\right) \sin \left(\frac{j \pi}{t_{1}}\right)\right] \\
& -\sum_{j=1}^{\infty} \mathrm{e}^{-2 c j t_{1}} f\left(2 j t_{1}+t\right) .
\end{aligned}
$$

for $0 \leq t \leq 2 t_{1}$. The above series (53) is called the Durbin formula and the last term of this series is called the discretization error. Honig and Hirdes (1984) developed a method for accelerating the convergence of the Fourier series and a procedure that computes that computes approximately the best choice of the free parameters.

\section{Numerical results and Discussion}

We have chosen the copper material for numerical computations. The physical data for which are given by Sherief et al. (2004)

$$
\begin{aligned}
& E=120 \mathrm{GPa}, v=0.34, T_{0}=0.293 \times 10^{3} \mathrm{~K}, \rho=8.954 \times 10^{3} \mathrm{Kg} \mathrm{m}^{-3}, K=0.386 \times 10^{3} \mathrm{Wm}^{-1} \mathrm{~K}^{-1}, \\
& \alpha_{t}=1.78 \times 10^{-5} \mathrm{~K}^{-1}, \alpha_{c}=1.98 \times 10^{-4} \mathrm{~m}^{3} \mathrm{Kg}^{-1}, c_{e}=0.3831 \times 10^{3} \mathrm{~J} \mathrm{Kg}^{-1} \mathrm{~K}^{-1}, \alpha=2.5 \mathrm{Kg} \mathrm{m} \mathrm{s}^{-2}, \\
& D=0.85 \times 10^{-8} \mathrm{Kg} \mathrm{s} \mathrm{m}^{-3}, a=1.02 \times 10^{4} \mathrm{~m}^{2} \mathrm{~s}^{-2} \mathrm{~K}^{-1}, b=9 \times 10^{5} \mathrm{Kg}^{-1} \mathrm{~m}^{5} \mathrm{~s}^{-2}, t=1.0 s, \tau_{0}=0.04 s, \\
& \tau_{1}=0.06 s, g_{0}=1, L=1, d=1, h=10, t_{0}=0.2 .
\end{aligned}
$$

Numerical computations have been carried out with the help of MATLAB software. By using this software, the displacement, lateral deflection, temperature change, mass concentration, axial stress and chemical potential for different value of time with respect to distance are computed numerically and shown graphically in Figs. 2-7. In all these Figs., solid line (-) corresponds to $t=1.4$, small dash line $(---)$ corresponds to $t=1.6$, solid line with centre symbol $(-*-)$ corresponds to $t=1.8$, and small dash line with centre symbol $(-*-*-*-)$ corresponds to $t=2.0$ respectively.

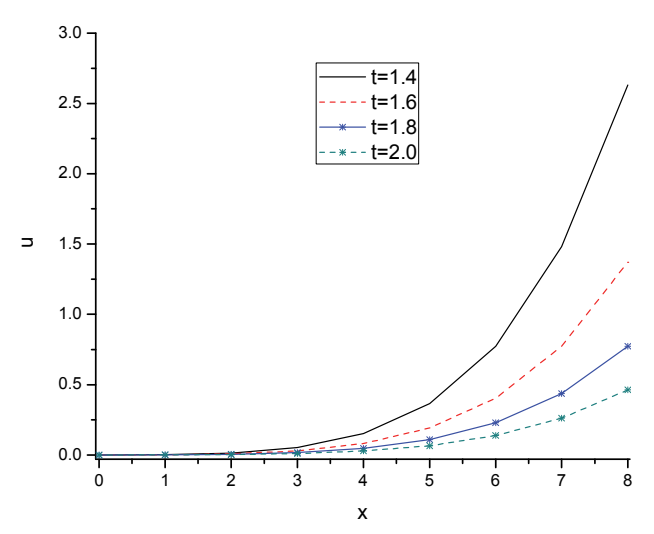

Fig. 2. Dispersion of displacement for different values of time

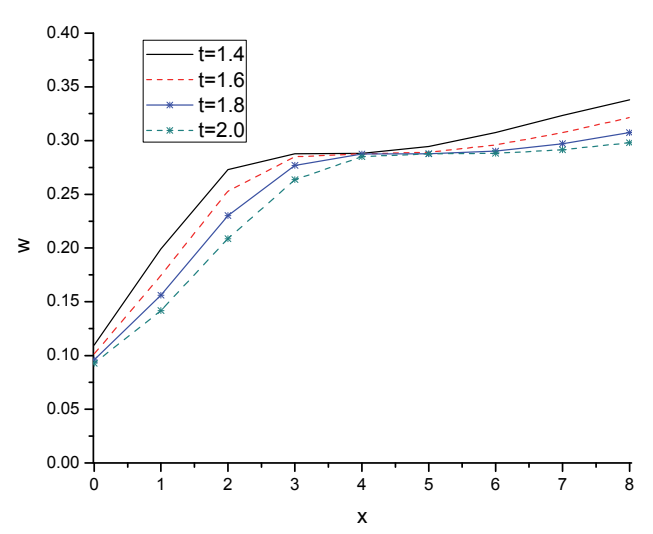

Fig. 3. Dispersion of lateral deflection for different values of time 


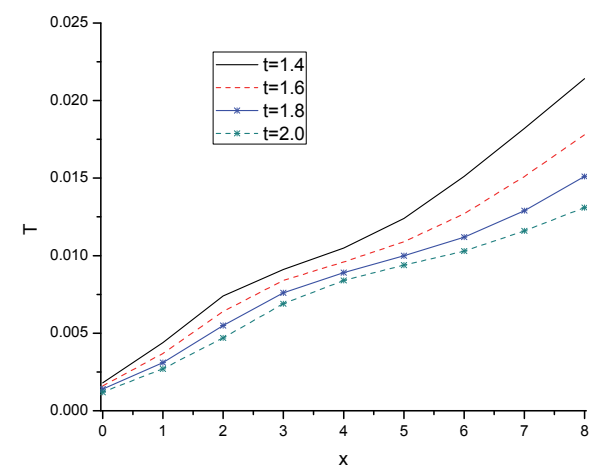

Fig. 4. Dispersion of temperature change for different values of time

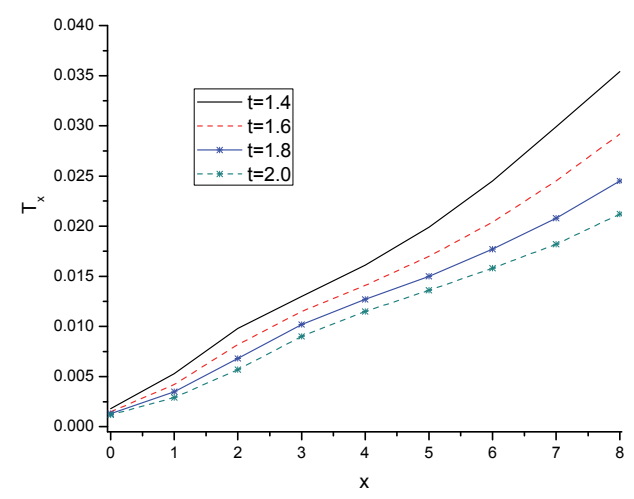

Fig. 6. Dispersion of axial stress for different values of time

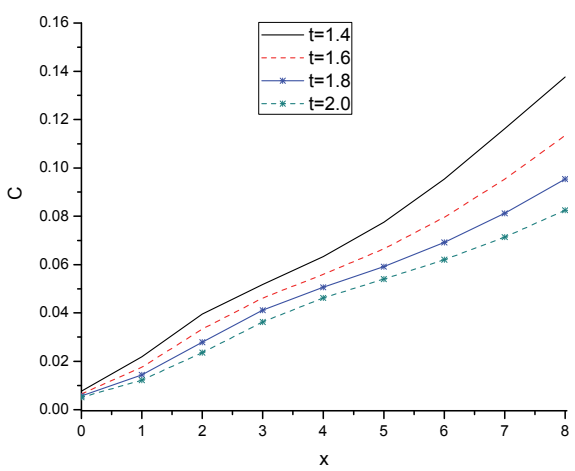

Fig. 5. Dispersion of mass concentration for different values of time

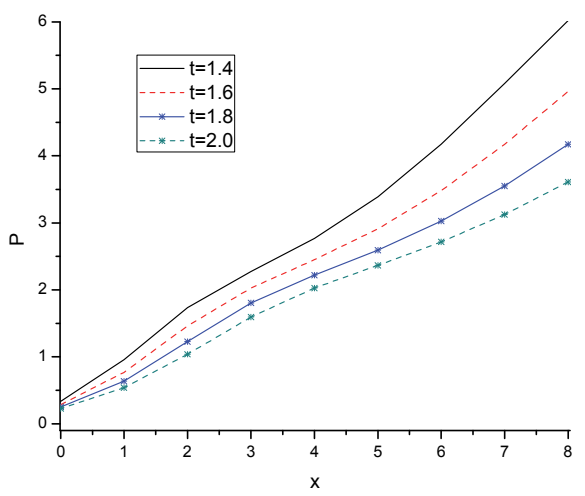

Fig. 7. Dispersion of chemical potential for different values of time

Fig. 2 represents the variation of displacement with respect to distance $x$ for different values of time. The value of displacement increases monotonically with the increase in time. It is noticed that the displacement decreases as time increases further. Fig. 3 illustrates the variation of lateral deflection with respect to distance for different values of time. It is noticed that the lateral deflection starts from maximum values which depends on time and attain minima at $x=4.5$ after that, magnitude of lateral deflection increases with the increase in distance to again attain maxima at the other end of the beam. Fig. 4 depicts the variation of temperature change through the length of the beam. It is seen that the behavior and variation of temperature change are similar in all cases. As time increases, the temperature change decreases with difference in their magnitude values. Fig. 5 shows the variation of mass concentration with respect to distance for different values of time. It is noticed that the values of mass concentration increases monotonically with the increase in time. Also, the value of mass concentration is more for $t=2 \mathrm{~s}$ and less for $t=1.4 \mathrm{~s}$ in the assumed region. Figs. 6 and 7 represent the variation of axial stress and chemical potential with respect to length of the beam for different values of time. The behavior and variation are same for both axial stress and chemical potential but there is significant difference between their values. As time increases, the values of axial stress and chemical potential decreases with increasing values of distance in the considered range.

\section{Conclusions}

In this study, the problem of generalized thermoelastic beam in modified couple stress theory with mass diffusion due to ramp type heating is investigated. A new model of thermoelastic nano-beam in the context of one relaxation time is developed. The Euler Bernoulli beam assumption and the Laplace 
transform technique are used to write the basic equations in the form of vector-matrix differential equation, which is then solved by eigenvalue approach. A numerical technique has been adopted to recover the solutions in the physical domain. The expressions for displacement, lateral deflection, temperature change, mass concentration, axial stress and chemical potential have been derived successfully and shown graphically for different values of time. It is observed from the figures that as time increases, the value of all physical quantities i.e. displacement, lateral deflection, temperature change, mass concentration, axial stress and chemical potential decreases but there is significant difference in their magnitude values. The method used in the present article is applicable to a wide range of problems in applied mathematics, science, engineering, geophysical and industrial sectors.

\section{References}

Abbas, I. A., Kumar, R., \& Rani, L. (2015). Thermoelastic interaction in a thermally conducting cubic crystal subjected to ramp-type heating. Applied Mathematics and Computation, 254, 360-369.

Abouelregal, A. E., \& Zenkour, A. M. (2014). Effect of phase lags on thermoelastic functionally graded microbeams subjected to ramp-type heating. Iranian Journal of Science and Technology. Transactions of Mechanical Engineering, 38(M2), 321.

Alzahrani, F. S., \& Abbas, I. A. (2016). Generalized thermoelastic diffusion in a nanoscale beam using eigenvalue approach. Acta Mechanica, 227(4), 955-968.

Chen, W., \& Wang, Y. (2016). A model of composite laminated Reddy plate of the global-local theory based on new modified couple-stress theory. Mechanics of Advanced Materials and Structures, 23(6), 636-651.

Choudhary, S., \& Deswal, S. (2010). Mechanical loads on a generalized thermoelastic medium with diffusion. Meccanica, 45(3), 401-413.

Cosserat, E. \& Cosserat, F. (1909). Theory of Deformable Bodies, Hermann et Fils, Paris.

Das, N. C., Lahiri, A., \& Giri, R. R. (1997). Eigenvalue approach to generalized thermoelasticity. Indian Journal of Pure and Applied Mathematics, 28, 1573-1594.

Green, A. E., \& Naghdi, P. M. (1993). Thermoelasticity without energy dissipation. Journal of Elasticity, 31(3), 189-208.

Honig, G., \& Hirdes, U. (1984). A method for the numerical inversion of Laplace transforms. Journal of Computational and Applied Mathematics, 10(1), 113-132.

Kumar, R., \& Devi, S. (2015). Interaction due to hall current and rotation in a modified couple stress elastic half-space due to ramp-type loading. Computational Methods in Science and Technology, 21(4), 229-240.

Kumar, R., \& Kansal, T. (2008). Propagation of Lamb waves in transversely isotropic thermoelastic diffusive plate. International Journal of Solids and Structures, 45(22), 5890-5913.

Kumar, R., Singh, R. \& Chadha, T. K. (2007). Eigenvalue approach to Micropolar thermoelasticity without energy dissipation. Indian Journal of Mathematics, 49(3), 355-369.

Mindlin, R. D., \& Tiersten, H. F. (1962). Effects of couple-stresses in linear elasticity. Archive for Rational Mechanics and Analysis, 11(1), 415-448.

Nowacki, W. (1974). Dynamical Problems of Thermo diffusion in Solids I. Bulletin of Polish Academy of Science and Technology, 22, 55-64.

Rao, S. S. (2007). Vibration of continuous systems. John Wiley \& Sons.

Reddy, J. N., Romanoff, J., \& Loya, J. A. (2016). Nonlinear finite element analysis of functionally graded circular plates with modified couple stress theory. European Journal of Mechanics-A/Solids, $56,92-104$.

Rezazadeh, G., Vahdat, A. S., Tayefeh-rezaei, S., \& Cetinkaya, C. (2012). Thermoelastic damping in a micro-beam resonator using modified couple stress theory. Acta Mechanica, 223(6), 1137-1152.

Samaei, A. T., Aliha, M. R. M., \& Mirsayar, M. M. (2015). Frequency analysis of a graphene sheet embedded in an elastic medium with consideration of small scale. Materials Physics and Mechanics, $22(2), 125-135$.

Sarkar, N., \& Lahiri, A. (2012). Eigenvalue approach to two-temperature magneto-thermoelasticity. Vietnam Journal of Mathematics, 40(1), 13-30. 
Sengupta, P. R. \& Ghosh, B. (1974a). Effect of couple stresses on surface waves in elastic media. Gerlands Beitr. Geophysik, Leipzig, 83, 309-318.

Sengupta, P. R., \& Ghosh, B. (1974b). Effect of couple-stresses on the propagation of waves in an elastic layer. Pure and Applied Geophysics, 112(2), 331-338.

Shaat, M., Mahmoud, F. F., Gao, X. L., \& Faheem, A. F. (2014). Size-dependent bending analysis of Kirchhoff nano-plates based on a modified couple-stress theory including surface effects. International Journal of Mechanical Sciences, 79, 31-37.

Sherief, H. H., \& Saleh, H. A. (2005). A half-space problem in the theory of generalized thermoelastic diffusion. International Journal of Solids and Structures, 42(15), 4484-4493.

Sherief, H. H., Hamza, F. A., \& Saleh, H. A. (2004). The theory of generalized thermoelastic diffusion. International Journal of Engineering Science, 42(5), 591-608.

Şimşek, M., \& Reddy, J. N. (2013). Bending and vibration of functionally graded microbeams using a new higher order beam theory and the modified couple stress theory. International Journal of Engineering Science, 64, 37-53.

Toupin, R. A. (1962). Elastic materials with couple-stresses. Archive for Rational Mechanics and Analysis, 11(1), 385-414.

Voigt, W. (1887). Theoretische Studien uber die Elasticitatsverhaltnisse der Krystalle Abh. Ges. Wiss. $p, 34$.

Wang, Y. G., Lin, W. H., \& Liu, N. (2015). Nonlinear bending and post-buckling of extensible microscale beams based on modified couple stress theory. Applied Mathematical Modelling, 39(1), 117-127.

Yang, F. A. C. M., Chong, A. C. M., Lam, D. C. C., \& Tong, P. (2002). Couple stress based strain gradient theory for elasticity. International Journal of Solids and Structures, 39(10), 2731-2743.

Zenkour, A. M., \& Abouelregal, A. E. (2016). Effect of ramp-type heating on the vibration of functionally graded microbeams without energy dissipation. Mechanics of Advanced Materials and Structures, 23(5), 529-537.

Zhang, J., \& Fu, Y. (2012). Pull-in analysis of electrically actuated viscoelastic microbeams based on a modified couple stress theory. Meccanica, 47(7), 1649-1658.

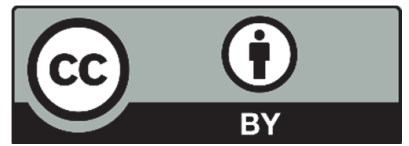

(C) 2017 by the authors; licensee Growing Science, Canada. This is an open access article distributed under the terms and conditions of the Creative Commons Attribution (CC-BY) license (http://creativecommons.org/licenses/by/4.0/). 